\title{
Metabolic surgery for the treatment of type 2 diabetes in obese individuals
}

\author{
David E. Cummings ${ }^{1,2} \cdot$ Francesco Rubino $^{3}$
}

Received: 13 February 2017 / Accepted: 4 October 2017 / Published online: 9 December 2017

(C) The Author(s) 2017. This article is an open access publication

\begin{abstract}
Several bariatric operations originally designed to promote weight loss have been found to powerfully treat type 2 diabetes, causing remission in most cases, through diverse mechanisms additional to the secondary consequences of weight loss. These observations have prompted consideration of such operations as 'metabolic surgery', used expressly to treat diabetes, including among patients who are only mildly obese or merely overweight. Large, long-term observational studies consistently demonstrate that bariatric/metabolic surgery is associated with reductions in all cardiovascular risk factors, actual cardiovascular events, microvascular diabetes complications, cancer and death. Numerous recent randomised clinical trials, directly comparing various surgical vs non-surgical interventions for diabetes, uniformly demonstrate the former to be superior for improvements in all glycaemic variables, as well as other metabolic endpoints. These benefits are similar among individuals with type 2 diabetes and a preoperative BMI of $30-35 \mathrm{~kg} / \mathrm{m}^{2}$ compared with traditional bariatric surgery patients with a BMI $>35 \mathrm{~kg} / \mathrm{m}^{2}$. The safety profiles of modern laparoscopic bariatric/metabolic operations are similar to those of elective laparoscopic hysterectomy and knee arthroplasty. However, more evidence regarding the risks, benefits and costs of surgery is needed from very long-term ( $>5$ year) randomised clinical trials powered to observe 'hard' clinical endpoints following the operations most commonly used today. Given the efficacy, safety and cost-effectiveness of metabolic surgery, the second Diabetes Surgery Summit (DSS-II) consensus conference recently placed surgery squarely within the overall diabetes treatment algorithm, recommending consideration of this approach for patients with inadequately controlled diabetes and a BMI as low as $30 \mathrm{~kg} / \mathrm{m}^{2}$, or $27.5 \mathrm{~kg} / \mathrm{m}^{2}$ for Asian individuals. These new guidelines have been formally ratified by 53 leading diabetes and surgery societies worldwide. Given this broad level of endorsement, we feel that the DSS-II recommendations should now replace the outdated National Institutes of Health (NIH) suggestions that have governed bariatric surgery practice and insurance compensation worldwide since 1991.
\end{abstract}

Keywords Bariatric surgery · Biliopancreatic diversion · Diabetes Surgery Summit · Gastric bypass · Ghrelin · Glucagon-like peptide-1 $\cdot$ Laparoscopic adjustable gastric banding $\cdot$ Metabolic surgery $\cdot$ Review $\cdot$ Vertical sleeve gastrectomy

\author{
Abbreviations \\ AHRQ Agency for Healthcare Research and Quality \\ BPD Biliopancreatic diversion \\ DSS-II Second Diabetes Surgery Summit
}

GLP-1 Glucagon-like peptide-1

LAGB Laparoscopic adjustable gastric banding

NIH National Institutes of Health

QALY Quality-adjusted life-year
Electronic supplementary material The online version of this article (https://doi.org/10.1007/s00125-017-4513-y) contains a slideset of the figures for download, which is available to authorised users.

\section{David E. Cummings}

davidec@u.washington.edu

Francesco Rubino

francesco.rubino@kcl.ac.uk

1 Department of Medicine, Division of Metabolism, Endocrinology and Nutrition, University of Washington, Box 358280 (mail stop 111), Seattle, WA 98195, USA
2 VA Puget Sound Health Care System, Seattle, WA, USA

3 Department of Surgery, Diabetes and Nutritional Sciences Division,
King's College London and King's College Hospital, 1st floor James
Black Centre, Denmark Hill Campus, 125 Coldharbour Road, London SE5 9NU, UK 
RYGB Roux-en-Y gastric bypass

SGLT Sodium-glucose cotransporter

VSG Vertical sleeve gastrectomy

\section{Introduction}

Type 2 diabetes is an expanding pandemic afflicting more than 400 million people, with estimates of 650 million cases by 2040. Despite ever-increasing options for pharmaceutical and lifestyle interventions, including medications recently shown to reduce cardiovascular events, many patients with diabetes fail to achieve glycaemic/metabolic treatment goals designed to reduce micro- and macrovascular complications. In the USA, only $52 \%$ of patients with type 2 diabetes maintain $\mathrm{HbA}_{1 \mathrm{c}}<53 \mathrm{mmol} / \mathrm{mol}(<7 \%)$, and only $19 \%$ reach this target along with $\mathrm{LDL}<5.6 \mathrm{mmol} / \mathrm{l}$ and blood pressure $<130$ / $80 \mathrm{mmHg}$, as recommended to minimise cardiovascular morbidity and mortality [1]. Implementing more effective strategies to prevent and treat diabetes has become a top priority in 21 st century medicine.

Recently, the second Diabetes Surgery Summit (DSS-II), an international consensus conference, developed global guidelines that recommend inclusion of bariatric/metabolic surgery among glucose-lowering interventions for selected patients with type 2 diabetes and obesity [2]. Endorsed thus far by 53 organisations worldwide, including major national diabetes and surgical societies, DSS-II guidelines were incorporated into the ADA Standards of Diabetes Care in 2017 [3]. This new guidance proposes that 'metabolic surgery' (involving procedures initially developed to treat obesity and dubbed 'bariatric surgery') should be considered as standard diabetes treatment options for appropriate candidates with inadequately controlled type 2 diabetes and a BMI $>30 \mathrm{~kg} / \mathrm{m}^{2}$, or $>27.5 \mathrm{~kg} / \mathrm{m}^{2}$ for Asian individuals. This conclusion is based on biological and clinical rationales. For example, mechanistic studies demonstrate that surgical manipulation of the gastrointestinal tract can exert powerful, beneficial effects on various facets of glucose homeostasis, independent of weight loss [4]. Moreover, a large body of clinical evidence, including numerous randomised clinical trials, documents that surgery improves blood glucose levels more effectively than any lifestyle and/or pharmaceutical intervention, often yielding longterm diabetes remission [5].

Inclusion of surgery among standard diabetes therapies represents a significant step forward in diabetes care and research. The mechanisms of metabolic surgery, albeit incompletely understood, underscore important roles for the gut in glucose homeostasis. Elucidating these mechanisms provides opportunities to clarify type 2 diabetes pathogenesis, potentially identifying targets for novel pharmacotherapeutics.

Leveraging insights provided by metabolic surgery, however, requires addressing practical and conceptual barriers, including widespread misconceptions about bariatric surgery. Despite compelling evidence of safety, efficacy and cost-effectiveness, using surgery as a diabetes intervention remained controversial until very recently.

Herein we review evidence regarding the effects of metabolic surgery in patients with obesity and type 2 diabetes, discussing the clinical, biological and economic rationales that support expanding its use as part of modern multidisciplinary approaches to diabetes care.

\section{Biological rationale for considering bariatric/metabolic surgery to treat type 2 diabetes}

It has become clear that certain operations initially designed to promote weight loss also powerfully improve glucose homeostasis, leading to type 2 diabetes remission in most cases, especially after procedures with intestinal bypass components [5]. Although approximately one-third of patients who initially achieve diabetes remission after Roux-en-Y gastric bypass (RYGB) later experience relapse, the median disease-free period for these individuals is 8.3 years [6]. Given the known benefits of tight vs standard glycaemic control in early diabetes on long-term cardiovascular disease ('legacy effect') [7, 8], even among people whose diabetes relapses several years after metabolic surgery, it is possible that this disease-free interval will also yield long-term cardiovascular benefits in relapsed individuals, especially in cases where initial diabetes duration is relatively short. Although this has not yet been proven in randomised clinical trials, very large, rigorously matched, non-randomised studies have shown that metabolic surgery is associated with long-term reductions in all cardiovascular risk factors, actual cardiovascular events, cancer and death [5, 9-12].

It is also clear that many diabetes-associated benefits of intestinal bypass operations, such as RYGB, result not only from known effects of weight loss on glucose homeostasis but also from diverse weight-independent glucose-lowering mechanisms. Several large bodies of evidence demonstrate this [13]. First, diabetes commonly remits very fast after surgery, before significant weight loss. Second, for a given amount of weight loss achieved with intestinal bypass surgery, larger improvements in glucose homeostasis and diabetes occur than with equivalent weight loss achieved by dieting, exercise or purely gastric-restrictive operations. Third, there is an inconsistent relationship between the amount of weight lost after intestinal bypass operations and the degree of diabetes remission, prevention and relapse after initial remission, as well as with rates of improvement in hard outcomes, such as heart attacks, strokes, cancer and death. Fourth, experimental operations and devices that replicate some of the intestinal physiology of metabolic operations, such as RYGB, without compromising 
gastric capacity, can powerfully improve or eliminate type 2 diabetes with little or no weight loss, disengaging the weightreducing and glucose-lowering effects of surgery. Finally, rare but illuminating cases of profound, late-onset hyperinsulinaemic hypoglycaemia (occurring 1-26 years postoperatively, typically at 2-4 years), sometimes requiring pancreatectomy, suggest long-term post-surgical stimulation of beta cell function and, possibly, mass. This latter point demonstrates that, occasionally, the effectiveness of surgery for the treatment of diabetes can be 'too powerful', something that would never occur with non-surgical weight loss.

A partial list of mechanisms mediating weight-independent glucose-lowering effects of gastrointestinal surgery is shown in the text box below. Although many of these specific mechanisms have only been demonstrated thus far in animals, compelling evidence indicates that metabolic surgery engages weight-independent glucose-lowering processes in humans [4]. This has important clinical implications because, although individuals with a lower BMI lose less weight postoperatively than do the severely obese, they still experience these weightindependent glucose-lowering effects. Accordingly, the benefits of bariatric/metabolic surgery for type 2 diabetes appear to be similar among people with a preoperative BMI $<35 \mathrm{~kg} / \mathrm{m}^{2}$ to those with a BMI $\geq 35 \mathrm{~kg} / \mathrm{m}^{2}$ [14], the traditional cut-off for bariatric surgery in patients with diabetes (see below). Admittedly, evidence for this assertion is limited for patients with a preoperative $\mathrm{BMI}<30 \mathrm{~kg} / \mathrm{m}^{2}$.

\section{Weight-independent glucose-lowering mechanisms of metabolic surgery}

\section{(1) Favourable changes in gut hormones}

a) Increased GLP-1 secretion and, hence, insulin (plus augmented postprandial peptide YY (PYY) and oxyntomodulin levels, contributing to weight loss)

b) In some cases, compromised secretion of the diabetes-promoting peptide ghrelin

2 Favourable changes in bile acid signalling

3 Increased glucose metabolism by the small intestine

4 Changes in intestinal nutrient sensing that improve insulin sensitivity, through both neural and humoral pathways

5 Reduced intestinal glucose transport via SGLT1

6 Reduced circulating branched-chain amino acids

7 Possible alterations in gut microbiota (although evidence is stronger for a role in weight loss than in improved glucose homeostasis)
The existence of weight-independent glucose-lowering mechanisms activated by traditional 'bariatric' operations, along with the profound clinical benefits of these procedures on type 2 diabetes (including among non-severely obese individuals), has prompted increasing consideration of surgery to treat diabetes per se as the primary intent, even among patients who are only mildly obese or merely overweight [15]. Hence, the term 'metabolic surgery' is becoming increasingly popular, and virtually every bariatric surgery society in the world has changed its name within the past several years to include the word 'metabolic'.

\section{Clinical rationale for considering metabolic surgery to treat type 2 diabetes}

Several large, long-term observational studies have uniformly shown that, compared with people receiving non-surgical care for obesity and diabetes, those who elect to undergo bariatric/ metabolic surgery demonstrate greater improvements in body weight, glycaemic control, diabetes remission, other cardiovascular risk factors, microvascular complications, heart attacks, strokes, cancer and death [5, 9-12]. Nine studies show reductions in all-cause mortality among patients who have undergone bariatric/metabolic surgery $[5,11,16,17]$, including a remarkable $92 \%$ decrease in diabetes-related deaths [17], and none have failed to observe this. Because these studies are not randomised clinical trials, however, potential unmeasured confounders are a concern. Perhaps people who elect to undergo a major intervention, such as surgery, are more motivated to maintain other healthy behaviours, such as changing their diet, exercising, ceasing smoking, seeing their doctors, taking medications, etc.

To address these concerns about unmeasured confounders in non-randomised studies, 11 recent randomised clinical trials have directly compared surgical vs non-surgical diabetes interventions. A DSS-II meta-analysis of these trials found almost universal results among them $[2,5]$. Compared with a wide variety of medical/lifestyle interventions, the four most commonly performed bariatric/metabolic operations (see below) consistently yielded superior improvements in body weight, all glycaemic measures (diabetes remission, glycaemic control, diabetes medication use, etc.), HDL and triacylglycerol levels, the metabolic syndrome, quality of life and overall medication use. These benefits were associated with acceptable complications and no surgical deaths to date (including follow-up of 1-5 years), among the 1050 surgical participants included. These findings represent nearly unanimous level 1a evidence from the metaanalysis of randomised clinical trials, including data on many patients with a preoperative BMI $<35 \mathrm{~kg} / \mathrm{m}^{2}$. Surgical patients also tended to experience greater improvements in LDL levels and hypertension than did non-surgical participants in these randomised clinical trials, although these differences were not as profound or consistent as the results for diabetes-related variables $[2,5,18]$. 


\section{Evidence for use of surgery to treat type 2 diabetes in patients with a BMI less than vs greater than $35 \mathrm{~kg} / \mathrm{m}^{2}$}

Traditionally, bariatric surgery is only permitted in people with type 2 diabetes if their BMI is $\geq 35 \mathrm{~kg} / \mathrm{m}^{2}$. This threshold is increasingly being challenged when considering metabolic surgery for diabetes. A recent meta-analysis of all published studies reporting on diabetes remission after bariatric/ metabolic surgery (including 94,579 surgical patients) showed that remission rates were equivalent in the 60 investigations in which mean baseline BMI of the study cohorts was $\geq 35 \mathrm{~kg} / \mathrm{m}^{2}$ and the 34 studies including participants with mean baseline BMI $<35 \mathrm{~kg} / \mathrm{m}^{2}$ (71\% vs $72 \%$, respectively) [19].

Similarly, the previously mentioned meta-analysis of 11 existing randomised clinical trials directly comparing various surgical vs non-surgical approaches to type 2 diabetes treatment found that the degree to which surgery out-performed medical/lifestyle interventions for diabetes remission and/or glycaemic control was equivalent among the five randomised clinical trials in which mean baseline BMI of the study cohorts was $<35 \mathrm{~kg} / \mathrm{m}^{2}$ compared with the six randomised clinical trials with mean participant baseline BMI $\geq 35 \mathrm{~kg} / \mathrm{m}^{2}$ (Fig. 1) [14].

Furthermore, according to a comprehensive comparative effectiveness analysis by the Agency for Healthcare Research and Quality (AHRQ), the safety of bariatric/metabolic surgery is at least as good for patients with a preoperative $\mathrm{BMI}<35 \mathrm{~kg} / \mathrm{m}^{2}$ as for those with baseline BMI $\geq 35 \mathrm{~kg} / \mathrm{m}^{2}$ [20].

The similar efficacy and safety of metabolic surgery to treat type 2 diabetes in patients with a preoperative BMI above vs below $35 \mathrm{~kg} / \mathrm{m}^{2}$ indicates that this arbitrary threshold, established 26 years ago by the National Institutes of Health (NIH) to determine surgical eligibility, is not well supported by extant evidence [21].

\section{Safety of metabolic surgery for type 2 diabetes}

Bariatric/metabolic surgery has become progressively safer over the past two decades, largely owing to the refinement of minimally invasive techniques. The vast majority of operations are now performed laparoscopically, with surgical mortality rates ten times less than for equivalent open operations [22]. Perioperative mortality rates from bariatric/metabolic surgery are less than those from laparoscopic cholecystectomy or appendectomy, and similar to elective laparoscopic hysterectomy and knee arthroplasty [23]. The perioperative complication rate for laparoscopic RYGB in a recent US National Registry is $3.4 \%$, which is less than that for laparoscopic hysterectomy, cholecystectomy or appendectomy [23].
All operations involve risk, and the most common longterm complications of bariatric/metabolic surgery are listed in the text box below [5]. Among the most frequent of these, iron deficiency can be chronic and sometimes requires repeated iron infusions. In addition, the incidence of less frequent but very serious adverse outcomes, for example suicide, accidental injuries and alcohol abuse, is substantially higher after some operations, with relative risk rates increasing by twoto threefold [5]. There is also considerable concern about long-term risks after certain procedures (especially those involving intestinal bypasses) on metabolic bone disease, osteoporosis and fracture rates. Definitive evidence regarding these hazards is incomplete.

Importantly, these potential complications must be weighed against possible adverse consequences of not having surgery, such as typically remaining obese, continuing to have diabetes and taking glucose-lowering medications (which can cause hypoglycaemia, weight gain, and other problems). As stated above, all nine existing published studies of overall long-term mortality among patients who have undergone bariatric/metabolic surgery have reported that death rates are reduced compared with those in non-surgical controls $[5,11$, 16, 17]. The aforementioned comprehensive AHRQ review of surgical vs non-surgical approaches to metabolic conditions, such as diabetes, concluded that adverse events of surgery were relatively low and that most surgical complications were minor and tended not to require major intervention [20].

\section{Economic rationale for considering metabolic surgery to treat type 2 diabetes}

Although only some studies show bariatric/metabolic surgery to be outright cost-saving, all studies find it cost-effective, with a price per quality-adjusted life-year (QALY) of $\sim$ US $\$ 5000-10,000$, well below the commonly accepted standard of US $\$ 50,000 /$ QALY for affordable healthcare

Most common long-term complications of bariatric/metabolic surgery

Iron deficiency (45-52\%)

Vitamin B12 deficiency (8-37\%)

Vitamin D 'deficiency' $(51 \%)$

Anaemia (15\%)

Calcium deficiency (10\%)

LAGB band slippage (15\% of LAGB procedures)

Re-operation (8\%)

All other individual complications $(<5 \%)$ 


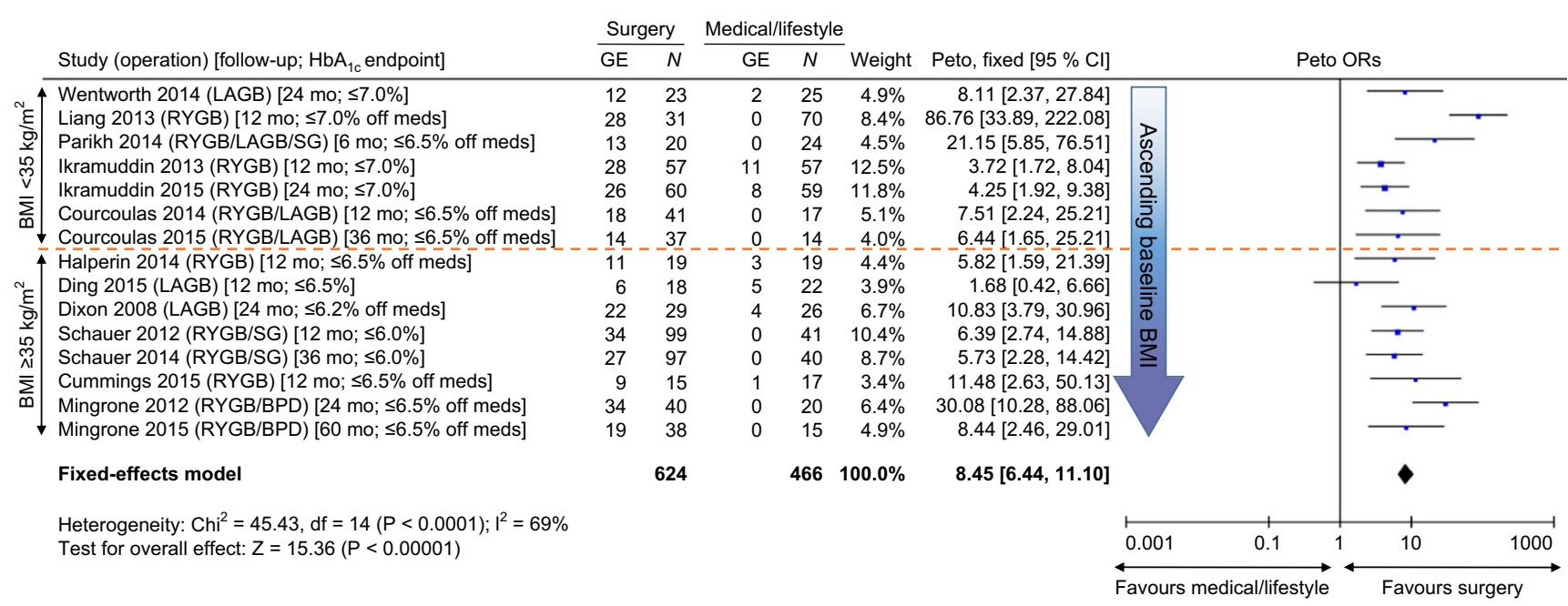

Fig. 1 Odds of diabetes remission or glycaemic control in all 11 randomised clinical trials of surgery vs medical/lifestyle care for type 2 diabetes. Forest plot of Peto ORs of primary outcomes (main glycaemic endpoints [GE], i.e. either diabetes remission or glycaemic control, depending on the trial) from each of the 11 published randomised clinical trials directly comparing bariatric/metabolic surgery vs medical/lifestyle treatments for diabetes. Data are arranged in order of ascending mean baseline BMI of each study group. The orange dotted line demarcates trials performed on cohorts with an average starting BMI either $<35 \mathrm{~kg} / \mathrm{m}^{2}$ or

interventions $[24,25]$. The cost-effectiveness of surgery is greater among people with diabetes than in those without it [25]. By comparison, as examples of non-surgical diabetes treatments, intensive medicinal glycaemic and lipid control cost US\$41,384/QALY and US\$51,889/QALY, respectively [26]. An important caveat is that most economic analyses of bariatric/metabolic surgery derive from modelling studies, rather than direct measurements within randomised clinical trials.

The time to return-on-investment for surgery varies widely among studies, from only a few years to as many as 9 years (or not observed within the period of follow-up). However, it is likely that the cost of bariatric/metabolic operations is fully repaid at some point after surgery if patients live long enough, from savings on medications not taken, hospitalisations avoided, complications not suffered, etc. Hence, insurance plans that typically retain patients for a long time (e.g. the Veterans Health Administration) should ultimately recoup the entire cost of surgery. Moreover, if all insurance plans covered bariatric/metabolic surgery, long-term healthcare costs for the entire system would decrease. Similar benefits would be observed in public healthcare systems, such as the UK National Health Service (NHS).

\section{Choosing the appropriate operation for type 2 diabetes}

Bariatric/metabolic operations that are currently in clinical use include RYGB (48\% of bariatric/metabolic surgery $\geq 35 \mathrm{~kg} / \mathrm{m}^{2}$. Column 1 depicts study duration and $\mathrm{HbA}_{1 \mathrm{c}}$ endpoint thresholds (in square brackets). Here, 'off meds' refers to a threshold achieved off all diabetes medicines, whereas otherwise the endpoints represent thresholds attained with or without such agents. ORs (shown with $95 \%$ CI) $>1$ indicate a positive effect of surgery compared with medical/lifestyle treatment. The pooled Peto OR $(95 \% \mathrm{CI})$ for all data was calculated using a fixed-effects model. mo, months; SG, sleeve gastrectomy. (C) 2016 by the ADA [14]. Adapted with permission from the ADA

worldwide), vertical sleeve gastrectomy (VSG, 42\%), laparoscopic adjustable gastric banding (LAGB, $8 \%$ ) and biliopancreatic diversion (BPD, 2\%). The order of effectiveness for weight loss and diabetes improvement is BPD $>$ RYGB $>$ VSG $>$ LAGB. The opposite order applies for safety.

By far, the most commonly used operations are RYGB and VSG, with VSG having overtaken RYGB in many nations. However, RYGB is more effective against diabetes and is considered by many as the gold standard operation for patients with this disease.

Novel bariatric/metabolic operations and devices are presently under development, including: (1) proximal intestinal bypass procedures for type 2 diabetes (e.g., duodenal-jejunal bypass surgery, endoscopically implanted endoluminal sleeves and endoscopic duodenal mucosal resurfacing); (2) ileal interposition surgery to enhance distal intestinal nutrient stimulation of L-cell peptide secretion; and (3) endoscopic techniques to reduce gastric volume for weight loss (e.g. new-generation gastric balloons, gastric plication and gastric electrical stimulation). Most of these approaches are currently used primarily in clinical trials.

\section{Evidence gaps in metabolic surgery research}

Although the mechanistic and clinical databases supporting a role for metabolic surgery in the overall diabetes treatment algorithm have burgeoned in recent years, including highly 
consistent level 1a evidence from randomised clinical trials directly comparing surgical vs non-surgical approaches, many key domains warrant additional research. Prominent among these are the following:

1. Long-term ( $>5$ year) results from randomised clinical trials regarding durability of glycaemic improvements are lacking. Efforts are underway to redress this gap, such as the Alliance of Randomized Trials of Medicine vs Metabolic Surgery in Type 2 Diabetes (ARMMS-T2D; ClinicalTrials.gov registration no. NCT02328599) consortium randomised clinical trial.

2. Longer-term data ( $>10$ years) are needed on efficacy and safety using non-randomised studies examining the operations currently performed. Although the superlative Swedish Obese Subjects (SOS) study database [9] is very large and extremely long term (and the most cited study of this nature in this field), it is primarily derived from observations in people with vertical-banded gastroplasty, which has not been used for decades, and LAGB, which is now seldom performed. Comparatively few patients underwent RYGB in this study and none had VSG, yet these two operations constitute $90 \%$ of bariatric/metabolic surgery currently performed.

3. Level 1 evidence is needed from randomised clinical trials sufficiently powered and of long enough duration to measure hard outcomes, such as microvascular and/or macrovascular events, cancer, death, etc.

4. More evidence is warranted regarding the long-term risks after intestinal bypass operations for metabolic bone disease, osteoporosis and fractures, especially from operations most commonly performed today.

5. We need true cost-effectiveness data from large randomised clinical trials, rather than just modelling methodologies.

6. Now that modern diabetes medications such as glucagonlike peptide-1 (GLP-1) agonists and sodium-glucose cotransporter (SGLT) 2 inhibitors have been shown to confer cardiovascular protection in large trials, these newer agents need to be directly compared against metabolic surgery in randomised clinical trials with 'hard' endpoints.

7. Level 1 evidence is needed regarding the long-term ( $>5$ year) rates of weight regain and diabetes recurrence after VSG, which is now the most commonly performed bariatric/metabolic operation in many nations.

8. It is likely that the combination of metabolic surgery and intensive medical/lifestyle treatment is the most effective strategy for diabetes control, and further research on the details of combining these approaches is warranted.

\section{New international guidelines for metabolic surgery to treat type 2 diabetes from the DSS-II}

In 2007, the first Diabetes Surgery Summit encouraged greater research on mechanisms of metabolic surgery as well as randomised clinical trials to define its role in type
Fig. 2 DSS-II: surgery in the type 2 diabetes treatment algorithm. Algorithm for the treatment of type 2 diabetes, including the option of bariatric/ metabolic surgery, as recommended by DSS-II voting delegates. Rx, treatment. (C) 2016 by the ADA [2]. Adapted with permission from the ADA

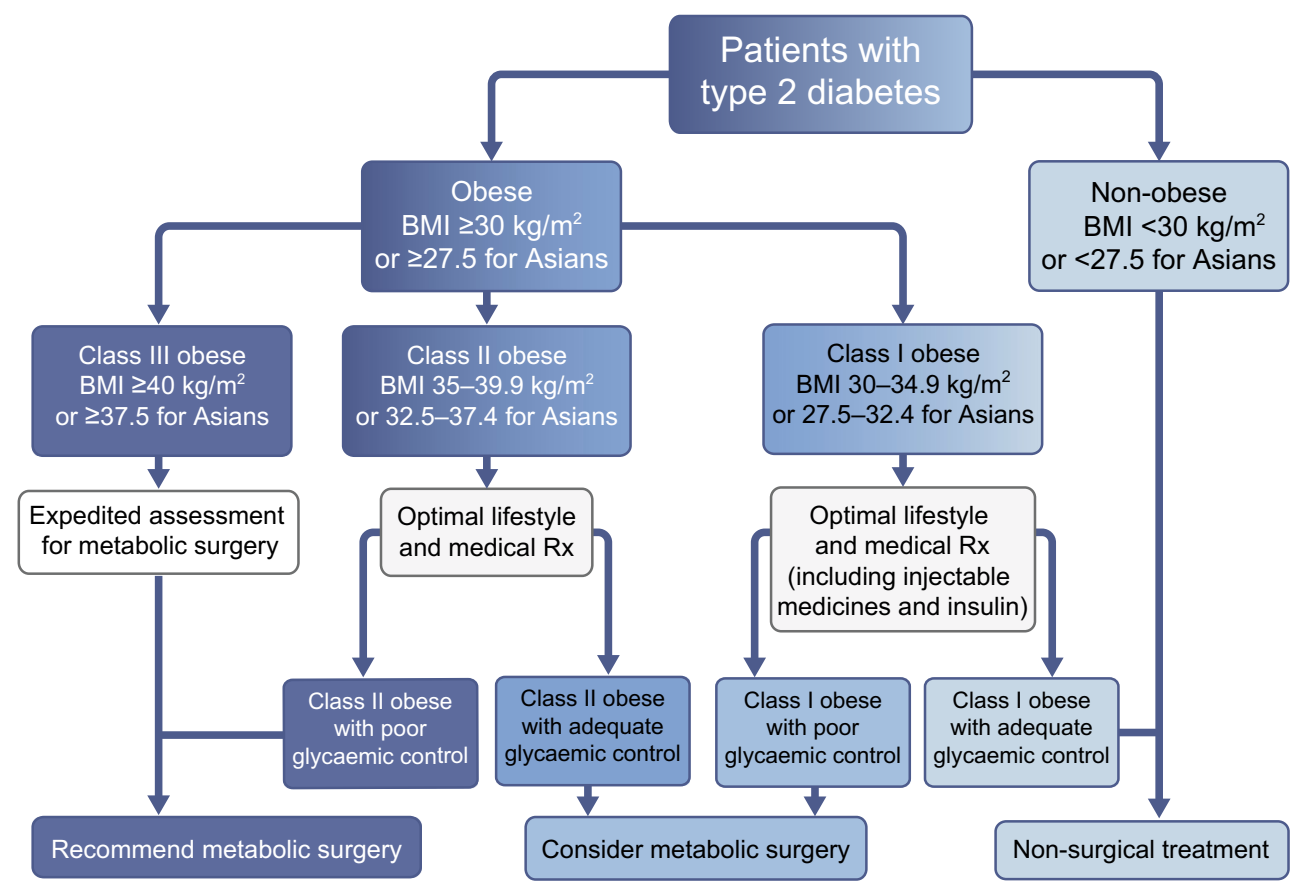


2 diabetes management [27, 28]. This helped generate funding to develop the evidence highlighted here. Based on this evidence, in 2015/2016 a panel of 48 international diabetes experts, representing numerous major worldwide diabetes societies, utilised a formal Delphi-like consensus-development process to generate new guidelines regarding the use and study of metabolic surgery for type 2 diabetes. Their recommendations were finalized at the DSS-II and, subsequently, codified in numerous related publications.

The 32 new DSS-II consensus statements and guidelines identify metabolic surgery as a standard option in the type 2 diabetes treatment algorithm (Fig. 2) [2]. With very high consensus, the delegates recommended that surgery should be considered to treat inadequately controlled type 2 diabetes in people with a BMI as low as $30 \mathrm{~kg} / \mathrm{m}^{2}$ (vs the prior NIH cut-off of $35 \mathrm{~kg} / \mathrm{m}^{2}$ ), or as low as $27.5 \mathrm{~kg} / \mathrm{m}^{2}$ in Asian patients. Although this change in BMI threshold is a modest numerical modification, it encompasses a very large number of people. In the USA, $-43 \%$ of individuals with diabetes have a BMI of $30-35 \mathrm{~kg} / \mathrm{m}^{2}$ [1] and, worldwide, the vast majority of people with diabetes have a BMI $<35 \mathrm{~kg} / \mathrm{m}^{2}$, including $>98 \%$ of East Asian individuals [29].

The DSS-II final recommendations have been formally ratified by 52 international societies thus far, including the ADA, the International Diabetes Federation (IDF), the Chinese Diabetes Society, Diabetes India, the European Association for the Study of Obesity (EASO), the Endocrine Society, the American Association of Clinical Endocrinologists (AACE), The Obesity Society and the American Gastroenterological Association (AGA), as well as the national diabetes organisations of many European and South American countries [2]. Hopefully, with this high degree of vetting and worldwide endorsement, these new guidelines will finally replace the seriously outdated 1991 NIH recommendations that have governed global practice and insurance compensation of bariatric surgery for more than a quarter of a century [21].

Funding DEC is supported by NIH grants R01 DK103842 and U01 DK114156.

Duality of interest DEC is a principal investigator on the Comparison of Surgery vs Medicine for Indian Diabetes (COSMID) randomised clinical trial, which is funded by Johnson \& Johnson, as well as the Alliance of Randomized Trials of Medicine vs Metabolic Surgery in Type 2 Diabetes (ARMMS-T2D) randomised clinical trial, which has previously been sponsored in part by Johnson \& Johnson plus Covidien, but is now fully funded by the NIH. FR declares that there is no duality of interest associated with this manuscript.

Contribution statement DEC drafted the manuscript, and both authors revised it critically for important intellectual content. Both authors approved the version to be published.
Open Access This article is distributed under the terms of the Creative Commons Attribution 4.0 International License (http:// creativecommons.org/licenses/by/4.0/), which permits unrestricted use, distribution, and reproduction in any medium, provided you give appropriate credit to the original author(s) and the source, provide a link to the Creative Commons license, and indicate if changes were made.

\section{References}

1. Ali MK, Bullard KM, Saaddine JB, Cowie CC, Imperatore G, Gregg EW (2013) Achievement of goals in U.S. diabetes care, 1999-2010. N Engl J Med 368:1613-1624

2. Rubino F, Nathan DM, Eckel RH et al (2016) Metabolic surgery in the treatment algorithm for type 2 diabetes: a joint statement by international diabetes organizations. Diabetes Care 39:861-877

3. American Diabetes Association (2017) Obesity management for the treatment of type 2 diabetes. Diabetes Care 40:S57-S63

4. Batterham RL, Cummings DE (2016) Mechanisms of diabetes improvement following bariatric/metabolic surgery. Diabetes Care 39: 893-901

5. Schauer PR, Mingrone G, Ikramuddin S, Wolfe B (2016) Clinical outcomes of metabolic surgery: efficacy of glycaemic control, weight loss, and remission of diabetes. Diabetes Care 39:902-911

6. Arterburn D, Bogart A, Sherwood N et al (2013) A multisite study of long-term remission and relapse of type 2 diabetes mellitus following gastric bypass. Obes Surg 23:93-102

7. Holman RR, Paul SK, Bethel MA et al (2008) 10-year follow-up of intensive glucose control in type 2 diabetes. N Engl J Med 359: $1577-1589$

8. Nathan DM, Cleary PA, Backlund JY et al (2005) Intensive diabetes treatment and cardiovascular disease in patients with type 1 diabetes. N Engl J Med 353:2643-2653

9. Sjostrom L (2013) Review of the key results from the Swedish Obese Subjects (SOS) trial - a prospective controlled intervention study of bariatric surgery. J Intern Med 273:219-234

10. Adams TD, Davidson LE, Litwin SE et al (2012) Health benefits of gastric bypass surgery after 6 years. JAMA 308:1122-1131

11. Arterburn DE, Olsen MK, Smith VA et al (2015) Association between bariatric surgery and long-term survival. JAMA 313:62-70

12. Adams TD, Arterburn DE, Nathan DM, Eckel RH (2016) Clinical outcomes of metabolic surgery: microvascular and macrovasular complications. Diabetes Care 39:912-923

13. Thaler JP, Cummings DE (2009) Hormonal and metabolic mechanisms of diabetes remission after gastrointestinal surgery. Endocrinology 150:2518-2525

14. Cummings DE, Cohen RV (2016) Bariatric/metabolic surgery to treat type 2 diabetes in patients with a BMI $<35 \mathrm{~kg} / \mathrm{m}^{2}$. Diabetes Care 39:924-933

15. Cummings DE, Cohen RV (2014) Beyond BMI: the need for new guidelines governing the use of bariatric and metabolic surgery. Lancet Diabetes Endocrinol 2:175-181

16. Sjostrom L, Narbro K, Sjostrom CD et al (2007) Effects of bariatric surgery on mortality in Swedish obese subjects. N Engl J Med 357: 741-752

17. Adams TD, Gress RE, Smith SC et al (2007) Long-term mortality after gastric bypass surgery. N Engl J Med 357:753-761

18. Vest AR, Heneghan HM, Agarwal S, Schauer PR, Young JB (2012) Bariatric surgery and cardiovascular outcomes: a systematic review. Heart 98:1763-1777

19. Panunzi S, De Gaetano A, Carnicelli A, Mingrone G (2015) Predictors of remission of diabetes mellitus in severely obese individuals undergoing bariatric surgery: do BMI or procedure choice matter? A meta-analysis. Ann Surg 261:459-467 
20. Maglione MA, Gibbons MM, Livhits M, et al (2013) Bariatric surgery and non-surgical therapy in adults with metabolic conditions and a body mass index of 30.0 to $34.9 \mathrm{~kg} / \mathrm{m} 2$. AHRQ Comparative Effectiveness Reviews, No. 82. Available from www.ncbi.nlm.nih.gov/books/NBK148685/. Accessed 9 October 2017

21. Consensus Development Conference Panel (1991) Gastrointestinal surgery for severe obesity. Ann Intern Med 115:956-961

22. Flum DR, Belle SH, King WC et al (2009) Perioperative safety in the longitudinal assessment of bariatric surgery. N Engl J Med 361: 445-454

23. Aminian A, Brethauer SA, Kirwan JP, Kashyap SR, Burguera B, Schauer PR (2015) How safe is metabolic/diabetes surgery? Diabetes Obes Metab 17:198-201

24. Picot J, Jones J, Colquitt JL et al (2009) The clinical effectiveness and cost-effectiveness of bariatric (weight loss) surgery for obesity: a systematic review and economic evaluation. Health Technol Assess 13:1-190
25. Keating C, Neovius M, Sjoholm K et al (2015) Health-care costs over 15 years after bariatric surgery for patients with different baseline glucose status: results from the Swedish Obese Subjects study. Lancet Diabetes Endocrinol 3:855-865

26. CDC Diabetes Cost-Effectiveness Group (2002) Cost-effectiveness of intensive glycaemic control, intensified hypertension control, and serum cholesterol level reduction for type 2 diabetes. JAMA 287:2542-2551

27. Rubino R, Kaplan LM, Schauer PR, Cummings DE (2010) The Diabetes Surgery Summit Consensus Conference: recommendations for the evaluation and use of gastrointestinal surgery to treat type 2 diabetes mellitus. Ann Surg 251:399-405

28. Rubino F, Schauer PR, Kaplan LM, Cummings DE (2010) Metabolic surgery to treat type 2 diabetes: clinical outcomes and mechanisms of action. Annu Rev Med 61:393-411

29. Lee WJ, Wang W, Lee YC, Huang MT, Ser KH, Chen JC (2008) Effect of laparoscopic mini-gastric bypass for type 2 diabetes mellitus: comparison of BMI $>35$ and $<35 \mathrm{~kg} / \mathrm{m}^{2}$. J Gastrointest Surg 12:945-952 